\title{
A review of accurate phase measurement methods and instruments for sinewave signals
}

\author{
Eulalia Balestrieri ${ }^{1}$, Luca De Vito ${ }^{1}$, Francesco Picariello ${ }^{1}$, Sergio Rapuano ${ }^{1}$, Ioan Tudosa ${ }^{1}$ \\ ${ }^{1}$ Department of Engineering, University of Sannio, Benevento, Italy
}

\section{ABSTRACT}

The phase measurement of sinewave signals is important in several applications, such as electric and electronic instrumentation; telecommunications; and optical interferometry. The uncertainty of the phase measurement has an essential role in ensuring the suitable performance of the devices and systems used by the relevant application. Some highly accurate phase measurement methods have been developed and implemented in different instrument types that are currently available on the market or have been proposed in the scientific literature, each capable of covering very different frequency ranges. This article presents an overview of these methods and instruments in order to highlight the characteristics in terms of the measurement uncertainty of the main methods and instruments that are used, by taking into account a varying operative frequency range. The standard deviations considered in the surveyed literature are used to identify a phase measurement method that is capable of covering a large high-frequency range, simultaneously maintaining a low value of measurement uncertainty, as requested by some applications (like waveform recorder frequency response testing).

\section{Section: RESEARCH PAPER}

Keywords: phase measurement; waveform recorders; wide frequency range; measurement uncertainty.

Citation: Eulalia Balestrieri, Luca De Vito, Francesco Picariello, Sergio Rapuano, loan Tudosa, A review of accurate phase measurement methods and instruments for sinewave signals, Acta IMEKO, vol. 9, no. 2, article 9, June 2020, identifier: IMEKO-ACTA-09 (2020)-02-09

Editor: Alexandru Salceanu, Technical University of Iasi, Romania

Received March 13, 2020; In final form April 23, 2020; Published June 2020

Copyright: This is an open-access article distributed under the terms of the Creative Commons Attribution 3.0 License, which permits unrestricted use, distribution, and reproduction in any medium, provided the original author and source are credited.

Corresponding author: Eulalia Balestrieri, e-mail: eubal@unisannio.it

\section{INTRODUCTION}

Phase measurement is crucial important information for characterising a sinewave signal. According to [1], 'the phase is the time (instant) for a given signal value on a waveform cycle'. Usually, the phase is an expression of the relative time difference between two corresponding features (peaks or zero crossings) of two waveforms with the same frequency [1]. In the case of a comparison between two waveforms, the phase difference or phase angle is typically expressed in degrees as a number greater than $-180^{\circ}$ and is less than or equal to $+180^{\circ}$ [1]. Moreover, phase can be sometimes expressed in radians, where one radian of a phase equals approximately $57.3^{\circ}$ [1].

Phase measurements are carried out in several applications for indirectly measuring strain, acceleration, and power quality as well as for characterising circuits and components in the frequency domain.

For example, in the case of optical interferometry, the desired measurand (e.g. strain, temperature, and surface deformation) is usually encoded in the phase of the signal obtained from the interference of two optical waves at the same frequency [2]. In radar applications, instead, the distance and the direction of a target, relative to the antenna, is estimated based on the phase of the electromagnetic wave reflected by the target itself [3]. In those and other indirect measurements, the uncertainty of the phase measurement has a crucial role in determining the measurement uncertainty of the derived quantities (e.g. strain and temperature).

Several electric and electronic instrumentation applications are particularly interested in the phase spectrum as the target measurand. For example, for monitoring the state of an electric power transmission network, the most widely used instrument is the Phasor Measurement Unit (PMU). An important task of a PMU is measuring the phase of each harmonic component of the voltage signal from the power supply network [4].

The phase spectrum is needed also for the estimation of the frequency response of a generic electrical circuit (Device Under Test [DUT]). In this case, the phase delay between a sinewave signal that is output by the DUT and the same sinewave that is input into the DUT is measured, [6].

A DUT that is particularly interesting from a research point of view is the waveform recorder (e.g. digital oscilloscope) for which the frequency response is essential in compensating for the systematic effects of the instrument on the voltage 
measurements [6]-[11]. The two main reasons for such interest are (i) the fact that it is a one-port DUT with an analog input and a digital output that cannot be easily and accurately compared to the input and (ii) the increasingly wide bandwidth of such DUTs, which makes it difficult to scan the whole phase spectrum. In [14], the authors proposed a method for phase delay measurement between the digitised output and the analog input of a waveform recorder, working up to $20 \mathrm{kHz}$. The authors declared that the method can be used for calibrating PMUs and digital low-power instrument transformers. However, the proposed method [6] is not designed for calibrating the current oscilloscopes available on the market working above $100 \mathrm{GHz}$ in of analog bandwidth. Although for the measurement of the frequency response of a high-frequency oscilloscope, the most commonly used technique is based on the evaluation of the pulse response of the oscilloscope under test, when moving to high frequencies, this technique does not maintain high accuracy. In that case, the most suitable technique is the sinewave sweep [8]. Knowing the phase of sinewave input into the waveform recorder, it is possible to estimate the phase response of the instrument for each input sinewave frequency [8], thereby providing the phase spectrum response of the waveform recorder.

To that end, it is necessary to measure the phase of the sinewave output by the source generator relative to a reference signal that is used for synchronising the clocks of the waveform recorder and the generator.

In all the cases mentioned above, the role of the measurement uncertainty associated with the phase measurement is essential for assuring the target uncertainty of the whole measurement system.

As quoted above, the methods and instruments developed to carry out high-accuracy phase measurements differ - in the output domain (time or frequency), the measurement frequency range, and the type of signal considered (electric or optical). The phase measurement methods and instruments are sometimes compared in terms of their capability of satisfying a specific application requirement, like the bandwidth of analysis, the measurement resolution, or the waveform shape. A comparison based on uncertainty, independent from the application, is currently missing.

In this article, therefore, a review of the main methods and instruments currently available on the market or described in the literature dealing with the phase measurement of sinusoidal signals is given, focusing on their measurement uncertainty performance and the covered frequency range. The presented analysis can be useful for helping researchers to find the method or the instrument they need by looking at the uncertainty and to pave the way to the design of a phase measurement method that is capable of covering a wide frequency range, maintaining low measurement uncertainty requirements.

This article is organised as follows. Firstly, the main phase measurement methods are described along with the instruments implementing them that are available on the market. Second, a comparison of the instruments previously described is carried out in terms of the expanded uncertainty for the phase measurement of the sinewave signals. Finally, conclusions are drawn.

\section{PHASE MEASUREMENT METHODS AND INSTRUMENTS}

Currently, different methods exist for measuring the phase delay of a sinusoidal signal relative to a reference signal. Most of the methods that have been proposed in the literature or in the instrumentation market as providing highly accurate phase measurements can be grouped into three main classes (see Table 1): (i) the event-counting-based method, (ii) the modulationbased method, and (iii) the sampling-based method. The first class provides the phase measurement as the time delay between two signals with the same frequency carried out by means of an event-counting technique. The instrument implementing this method is the universal counter, and the operating frequency order of magnitude usually ranges from $10^{-3} \mathrm{~Hz}$ to $10^{8} \mathrm{~Hz}$.

The modulation-based methods provide the phase delay introduced by a DUT, using a frequency mixer to multiply the sinewave under test with a signal from a reference oscillator. This method is used by three different kinds of instruments: the Vector Signal Analyser (VNA), the Phase-to-Voltage Converter (PVC), and the interferometer. Both the VNA and PVC operate on electric signals, while the interferometer on optical signals. Their output domain is the frequency one. The frequency ranges covered by the three instruments are different; for example, from $10^{3} \mathrm{~Hz}$ to $10^{11} \mathrm{~Hz}$ for the VNA, from $10 \mathrm{~Hz}$ to $10^{3} \mathrm{~Hz}$ for the PVC, and from $10^{11} \mathrm{~Hz}$ to $10^{12} \mathrm{~Hz}$ for the interferometer.

Finally, the sampling-based methods are based on sampling the input sinewave using a reference clock, digitising it and processing it with algorithms that estimate the unknown phase from the sample record. A typical instrument implementing a sampling-based method is the PMU. The operating frequency range, in this case, is from $10 \mathrm{~Hz}$ to $10^{2} \mathrm{~Hz}$.

In the following subsections, the three classes of phase measurement methods along with the typical instruments implementing them are described, highlighting their main advantages and limitations. Although in time and frequency metrology, the phase difference is usually stated in units of time [1], in this article, its value and measurement uncertainty are expressed in degrees, since this is the way they are expressed in the datasheets and in the considered scientific articles. However,

Table 1. Classification of the main phase measurement methods belonging to the event-counting-based, modulation-based, and sampling-based method class available in the literature and commercial instruments.

\begin{tabular}{|c|c|c|c|c|c|c|}
\hline \multirow[t]{2}{*}{ Method } & \multirow[t]{2}{*}{ Instrument } & \multicolumn{2}{|c|}{$\begin{array}{l}\text { Frequency range } \\
\text { in } \mathrm{Hz}\end{array}$} & \multirow[t]{2}{*}{ Type of measurement } & \multirow[t]{2}{*}{ Type of signal } & \multirow[t]{2}{*}{ Output domain } \\
\hline & & Min. & Max. & & & \\
\hline Event-counting-based & Universal counter & $10^{-3}$ & $10^{8}$ & $\begin{array}{l}\text { Phase delay between two } \\
\text { isofrequential signals }\end{array}$ & Electric & Time \\
\hline Modulation-based & $\begin{array}{l}\text { Vector network analyser (VNA) } \\
\text { Phase-to-voltage converter (PVC) } \\
\text { Interferometer }\end{array}$ & $\begin{array}{l}10^{3} \\
10 \\
10^{11}\end{array}$ & $\begin{array}{l}10^{11} \\
10^{3} \\
10^{12}\end{array}$ & $\begin{array}{l}\text { Phase delay introduced by a } \\
\text { device under test (DUT) }\end{array}$ & $\begin{array}{l}\text { Electric } \\
\text { Optical }\end{array}$ & Frequency \\
\hline Sampling-based & Phasor measurement unit (PMU) & 10 & $10^{2}$ & $\begin{array}{l}\text { Phase of a sinewave signal } \\
\text { according to a reference clock }\end{array}$ & Electric & Time \\
\hline
\end{tabular}




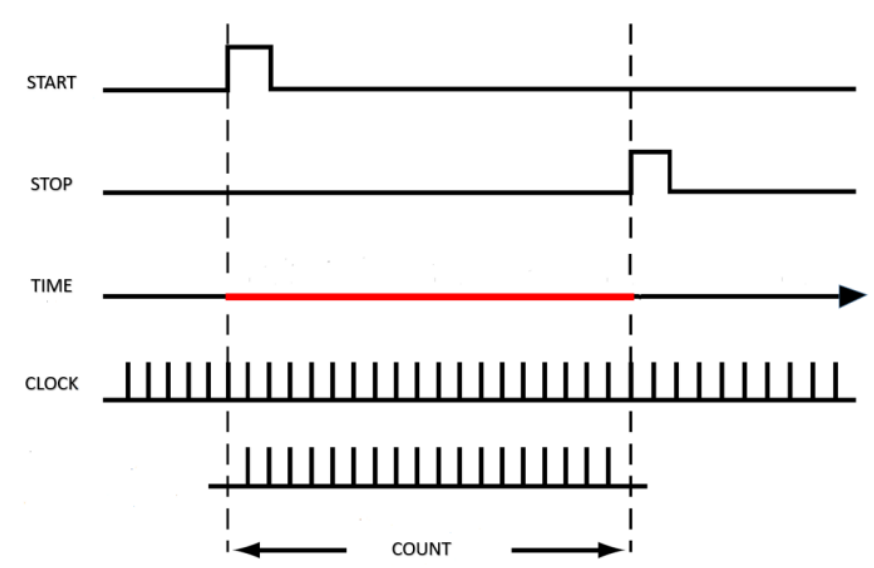

Figure 1 . The principle of operation of the event-counting-based method considering the positive-going transition to define the start and stop signals of the count.

the degrees can be easily converted in units of time, considering that the time interval for $1^{\circ}$ of the phase is inversely proportional to the frequency. Therefore, if the frequency of a signal is given by $f$, then the time $t_{\operatorname{deg}}$ (in seconds) corresponding to $1^{\circ}$ of the phase is $t_{\mathrm{deg}}=1 /(360 f)=T / 360$, [1] where $T$ is the period of the signal.

\subsection{Event-counting-based methods}

The methods based on event counting provide phase delay measurements between two isofrequential electrical signals.

In particular, the two signals are converted into square waves using a Schmitt trigger circuit.

The first positive-going or negative-going transition of one square wave is used as a start signal of a counter. The stop signal is generated at the first positive-going or negative-going transition of the other square wave. The counter measures the number of transitions of an internal clock signal between the start and the stop events (see Figure 1). Based on the number of clock signal transitions and the period of the internal clock, the time delay is measured. Then, by measuring the frequency of the signals under test, the phase delay is estimated.

An example of a time interval counter for measuring phase delay between two atomic clocks is proposed in [12]. This counter has an internal clock frequency of $400 \mathrm{MHz}$, which corresponds to an absolute time resolution of $2.5 \mathrm{~ns}$.

An example of high-performance universal counter that is available on the market [13] has a frequency range from $1 \mathrm{mHz}$ up to $350 \mathrm{MHz}$ with a time resolution of $20 \mathrm{ps}$, and an uncertainty value in the order of $0.1^{\circ}$ from $1 \mathrm{mHz}$ up to $100 \mathrm{kHz}$. For frequency values between $100 \mathrm{kHz}$ and $350 \mathrm{MHz}$, the uncertainty value as a quadratic function of frequency varies [13].

The main disadvantages of the counting-based method are (i) that the noise on the input signals causes time-varying transition occurrence instants of the square waves generated by the Schmitt trigger circuits and (ii) that the measurement accuracy is mainly affected by the time resolution and stability of the reference internal clock.

\subsection{Modulation-based methods}

The modulation-based methods for phase measurement use a frequency mixer to multiply the sinewave under test with a signal from a reference oscillator. The mixer output is filtered by a low-pass or band-pass filter and is subsequently acquired with an Analog-to-Digital Converter (ADC).

The following instruments exploit this method: (i) the VNA, (ii) the PVC circuit, and (iii) interferometers.

The VNA is the most widely used instrument for characterising the frequency response of circuits or systems in terms of phase and magnitude spectrum. In the VNA measurements, a sinewave signal generated internally stimulates the DUT, and two or more receivers are used for measuring the phase and amplitude of the incident and reflected waves at the DUT input and output ports. The VNA has, as a reference oscillator, one Local Oscillator (LO) per port. Each receiver typically has two mixers, one to multiply the LO output with the incident signal and the other to multiply the LO output with the received (reflected or transmitted) signal. An ADC acquires the incident, reflected, or transmitted wave amplitude. The ADCs are synchronised, and the acquired samples are used for estimating the phase delays between the reflected and incident waves.

This instrument can be used for measuring the phase delay of a DUT with at least one input and one output port, which is not the case for signal generators or waveform recorders, which have only one input port. VNAs, however, can be used to measure the complex input impedance of a waveform recorder input port.

In [14], the measurement specifications of a commercial VNA are reported. This instrument exhibits an uncertainty value in the order of $0.01^{\circ}$ for a frequency range of $900 \mathrm{~Hz}$ to $120 \mathrm{GHz}$.

The PVC is based on the multiplication of the sinewave under test with a sinewave of the same frequency. The resultant signal has a DC component that is proportional to the phase delay between the sinewaves. In the case of a phase delay that is equal to $90^{\circ}$, the output DC component is ideally equal to zero. It can be used for measuring the phase delay between two sinewave signals or the phase delay introduced by a system with at least two ports.

In [15], the authors present a high-performance automatic fully-analog PVC circuit for the phase shift measurements of periodic waveforms based on that method, working in a frequency range of around $100 \mathrm{~Hz}$. In particular, the PVC has been tested for measuring the phase of electrical signals, and the authors assessed the measurement uncertainty to be in the order of $0.001^{\circ}$.

Apart from the narrow frequency range, the main drawback of PVCs is that the amplitude of the DC component is affected by the difference between the frequencies of both signals used as mixer inputs. For this reason, a Phased-Locked-Loop (PLL) circuit is used to control the frequency of the reference source signal so that it is nominally equal to the frequency of the sinewave under test. Of course, the measurement accuracy depends on the PLL stability [16].

At terahertz frequencies, the modulation-based method is often implemented by means of interference between optical and electrical signals [17]. In this case, the aim can be to measure the delay introduced by a sample material on a reference signal. The systems used for performing these measurements (Figure 2) are (i) the conventional self-heterodyne system and (ii) the balanced self-heterodyne system. For both systems, a continuous electrical $\mathrm{THz}$ wave is generated by means of an optical-to-electrical conversion, also referred to as photomixing [18].

In particular, two frequency-detuned free-running lasers operating at two different frequencies, $f_{1}$ and $f_{2}$, are combined to produce a beat note at the frequency $f_{2}-f_{1}$. The frequency of Laser1 is coherently shifted (by $f_{s}$ ) with an optical Frequency Shifter (FS) so that the Radio-Frequency (RF) and optical LO 


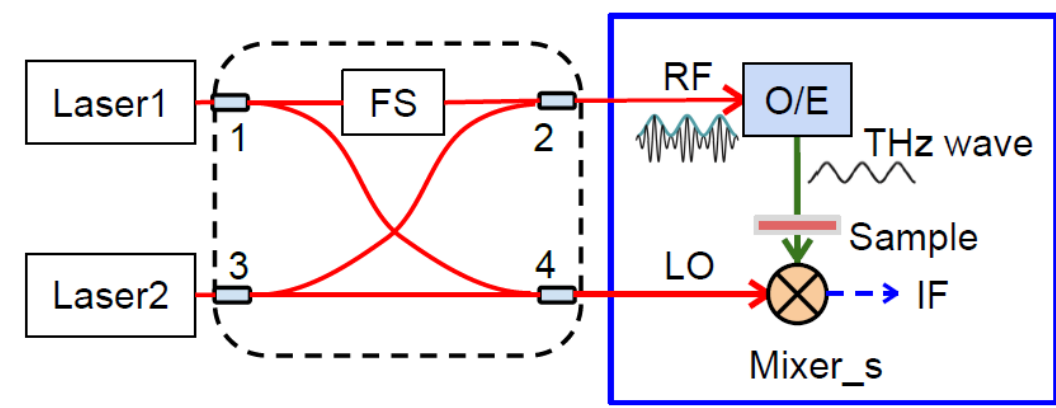

Conventional

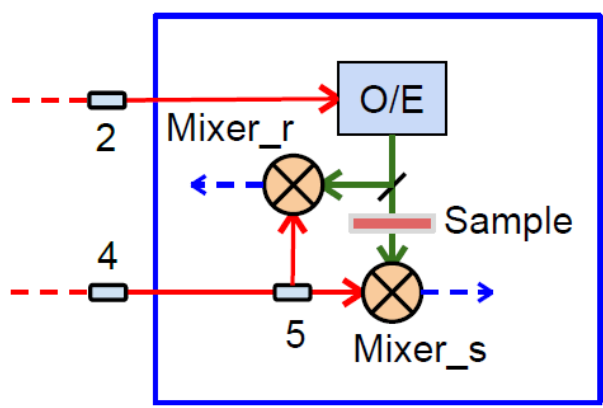

Balanced

(a)

(b)

Figure 2. Configurations of the (a) conventional and (b) balanced self-heterodyne system. FS $=$ optical frequency shifter and $\mathrm{O} / \mathrm{E}=$ optical-to-electrical converter [17]. Nodes 1 and 3 are optical splitters, and nodes 2, 4 and 5 are optical combiners.

signals have the same frequency/phase noise at the different average frequencies, realising the self-heterodyne detection [18][19] (Figure 2a). The RF frequency can be tuned by tuning the frequency of one of the lasers - without changing the IF frequency $\left(f_{s}\right)$ [19].

The RF signal is transmitted to the sample under test and the provided output mixed with the reference optical beat signal, LO.

The phase and the amplitude of the output signal provided by the mixer can be measured simultaneously by means of a twophase lock-in amplifier [20]. In the self-heterodyne system, the frequency fluctuation of the RF signal and that of the optical LO signal has the same property; thus, the frequency fluctuations of the free-running lasers are cancelled out by means of the mixing operation [21]. However, the conventional self-heterodyne system is affected by phase instabilities or phase drift due to the optical phase fluctuations imposed in the optical fibers. In the balanced self-heterodyne system, this problem is resolved by dividing the $\mathrm{THz}$ wave into two paths (Figure 2b). Each amplitude and phase detected by two mixers is measured by a two-channel dual-phase lock-in amplifier. After the detection, the common phase noises are subtracted [17][21].

In this case, the authors of [17] identified an uncertainty in the phase measurements in the order of $1^{\circ}$ at $300 \mathrm{GHz}$.

\subsection{Sampling-based methods}

The phase measurement methods based on sampling techniques have in common the direct digitisation of the signal from the source under test without modulating it. Digital signal processing algorithms are then used to estimate the unknown phase from the sample record. In particular, for estimating the phase of a sinewave signal, a sine fit can be performed on the acquired samples. To that end, the ADC used for sampling and digitisation must have sufficient amplitude resolution, such that the quantisation noise is lower than the noise from the measurement circuit.

An example of an instrument based on the sampling method is the PMU. In [4], the authors present a PMU for the measurement of phasors in three-phase power distribution networks. The proposed PMU consists of an ADC for acquiring four analog signals, the three monitored electrical inputs (voltages or currents) and the synchronisation signal provided by a GPS receiver. Then, the measurements of the amplitude and phase of the harmonic components in the three-phase system are provided by means of a Fourier analysis of the acquired samples. Such a method requires a significant number of samples to be acquired, in the order of $10^{3}$, to achieve accurate (less than $0.01 \%$ of actual) amplitude and phase measurements. For this reason, this method is useful for the accurate measurement of the phase of sinewaves at low frequencies (order of $100 \mathrm{~Hz}$ ). In [22], the authors report uncertainty in the order of $0.0001^{\circ}$ at $50 \mathrm{~Hz}$.

A small number of samples can be used by applying interpolation methods for pure sinewave signals. For example, if the sinewave frequency is known, it is possible to use a threeparameter sine fit [23] to estimate the phase of the signal. Conversely, if the frequency is unknown, a four-parameter leastsquares sine fit can be used. In all the above cases, the measurement uncertainty is affected by (i) the noise on the signal under test, (ii) the aperture uncertainty of the sampling circuit, and (iii) the jitter on the sampling clock.

The equivalent-time sampling techniques, which are used in some high bandwidth oscilloscopes, acquire each sample of a periodic waveform at a given delay (instant) after a trigger signal. A sample is acquired by the oscilloscope if the trigger signal exceeds a user-defined threshold. One sample per waveform instant is acquired per trigger event, and many samples per instant and many instants per waveform are necessary to capture a waveform. As reported in [8], the jitter in the time-base circuitry and between the trigger event and each acquired sample (i.e. trigger jitter [24]) can affect the timing and shape of the displayed waveform. The jitter trigger effectively acts to impose a low-pass filter on the acquired waveform. Furthermore, if the input signal, which is used for generating the trigger event, has a slow slew rate (low frequency signal) or a low Signal-to-Noise Ratio (SNR), the trigger jitter increases [27].

\subsection{Application limits of the event-counting-, modulation-, and sampling-based methods}

The main limitations of the described phase measurement methods can be summarised as follows: (i) the phase measurement is obtained using a reference signal that has the same frequency of the sinewave signal (the event-counting-based and modulation-based methods), and/or (ii) the phase measurement is provided for a limited frequency range (interferometric and sampling methods), and/or a huge number of signal samples per period is required (sampling methods) [4][22]. 


\section{PHASE MEASUREMENT UNCERTAINTY COMPARISON}

In this section, a comparison of the instruments described in the previous section in terms of the expanded uncertainty for the phase measurement of the sinewave signal is proposed. In the following section, the analysis of the data from the comparison is described first, and a discussion on the obtained results is then provided.

\subsection{Data analysis}

Whenever a commercial instrument is available to measure phase, the comparison is carried out using the instrument characteristics that are stated by the manufacturer. When the references are technical papers, the standard deviations of the published results are used.

Five instruments or methods are compared: one, a universal counter, belongs to the event-counting-based methods; three, a PVC, an interferometric technique, and a VNA, belong to the modulation-based methods; and one, an ADC-based technique, belongs to the sampling-based methods.

In particular, the instruments or methods considered are: (i) a universal counter [13] with a frequency range of $1 \mathrm{mHz}$ to $350 \mathrm{MHz}$, (ii) the PVC, working at very low frequencies, $7 \mathrm{~Hz}$ to $770 \mathrm{~Hz}$ [15], (iii) an interferometric technique at frequencies in the order of $\mathrm{THz}$ [15], (iv) the technique based on ADC and three-parameter sine-fit [22], used at very low frequencies (order of $\mathrm{Hz}$ ), and (v) a VNA, working in the frequency range of $500 \mathrm{~Hz}$ to $125 \mathrm{GHz}$ [14].

For the expression of the Type B combined standard uncertainty values, $u_{\Phi}$, related to the universal counter, the following formula provided by the manufacturer has been used [13]:

$$
\begin{gathered}
u_{\Phi}=\left[3 \cdot \sqrt{2\left(T_{\mathrm{SS}}^{2}+T_{\mathrm{E}}^{2}\right)}+T_{\mathrm{LTE}}+\text { skew }+2\right. \\
\left.\cdot T_{\text {accuracy }}\right] \cdot f_{\mathrm{src}} \cdot 360\left[^{\circ}\right],
\end{gathered}
$$

Where $T_{\mathrm{SS}}$ is the timing resolution of a start/stop measurement event, $T_{\mathrm{E}}$ is the threshold error due to the input signal random jitter, $T_{\text {LTE }}$ is the timing error due to the threshold level, skew is the additional time error if two channels are used for a measurement, $T_{\text {accuracy }}$ is the measurement error between two points in time, and $f_{\text {src }}$ is the frequency of the signal under test, which is a sinewave. According to the Keysight 53200A Series $\mathrm{RF} /$ universal frequency counter/timers datasheet [12], the following values have been associated with each component of the uncertainty equation: $T_{\mathrm{SS}}=20 \mathrm{ps}, T_{\mathrm{E}}=0 \mathrm{ps}$, skew $=50 \mathrm{ps}$, $T_{\text {accuracy }}=100$ ps. Furthermore, $T_{\text {LTE }}$ has been evaluated according to the equation:

$$
T_{\mathrm{LTE}}=\frac{T_{\mathrm{LSE}-\text { start }}}{S R_{- \text {start }}}+\frac{T_{\text {LSE-stop }}}{S R_{- \text {stop }}},
$$

where $T_{\mathrm{LSE}-\mathrm{x}}$ is the threshold setting error given by:

$$
T_{\mathrm{LSE}-\mathrm{x}}=0.002 \cdot \text { setting }+0.001 \cdot \text { range, }
$$

where, setting $=0 \mathrm{~V}$ and range $=5 \mathrm{~V}$, and $S R_{-x}=2 \cdot \pi \cdot f_{\text {src }}$.

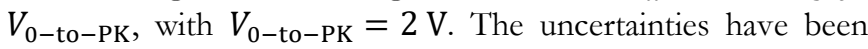
evaluated in the measurement frequency range of the universal counter, from $1 \mathrm{mHz}$ up to $350 \mathrm{MHz}$ and have been plotted in orange in Figure 3.

The PVC system considered in the comparison, described in [15], has been designed for working in the frequency range of a few $\mathrm{mHz}$ to $100 \mathrm{kHz}$. Furthermore, the experimental results, in terms of standard uncertainty on phase measurements of $0.01^{\circ}$,

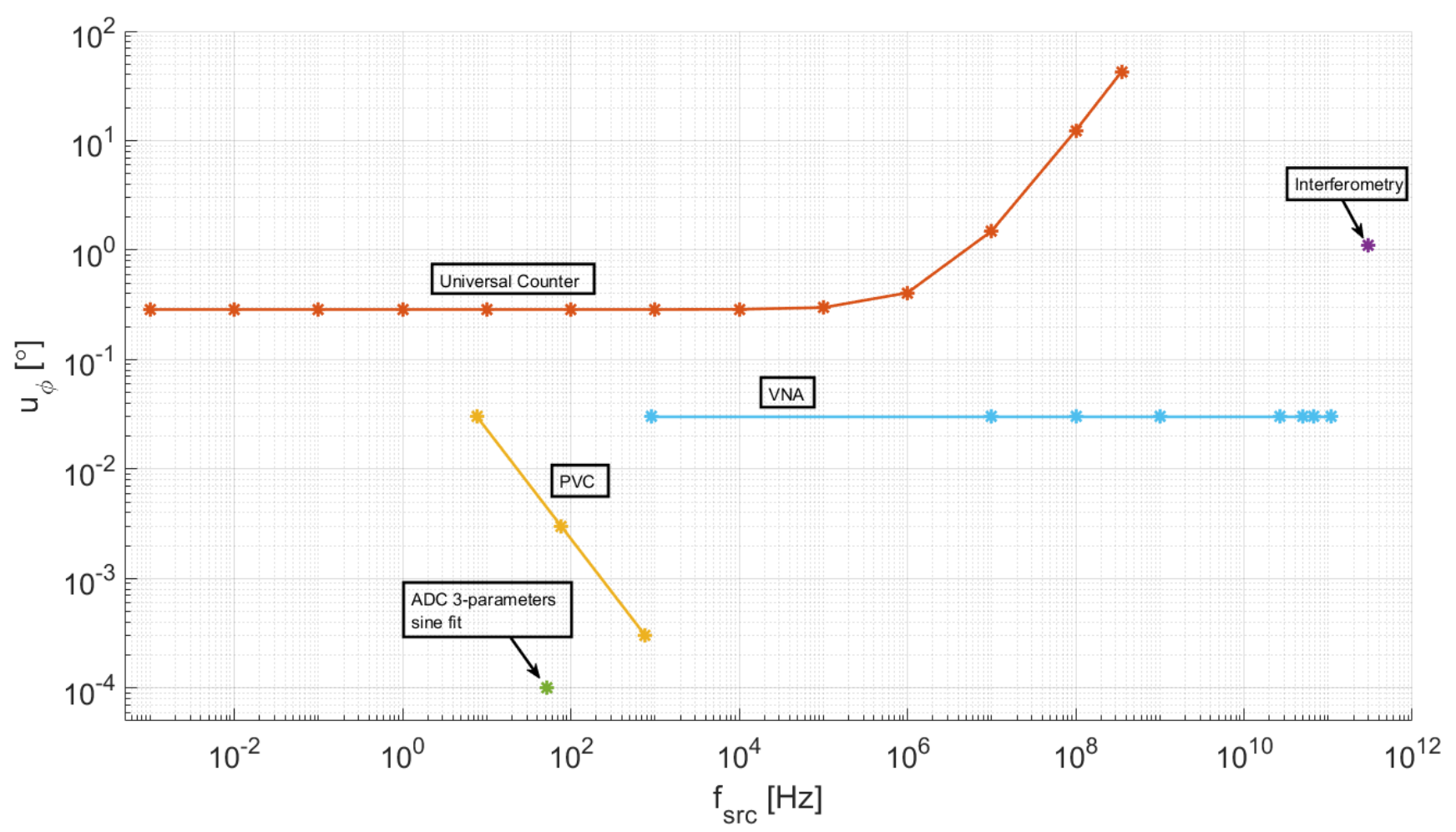

Figure 3. Performance comparison in terms of the expanded uncertainty of the instruments available on the market for the measurement of the phase of sinewave signals. 
$0.001^{\circ}$, and $0.0001^{\circ}$, at the frequency values of $7.7 \mathrm{~Hz}, 77 \mathrm{~Hz}$, and $770 \mathrm{~Hz}$, respectively, are reported and plotted in yellow in Figure 3.

The modulation method based on the interferometric technique at $\mathrm{THz}$ frequencies for characterising sample materials, described in [15], is aimed at measuring the phase delay introduced by the sample material, which is driven by an electrical signal. The proposed prototype exhibits an expanded uncertainty on phase measurements of $0.37^{\circ}$ at $300 \mathrm{GHz}$ [15], reported with the purple dot in Figure 3.

The technique based on sine fitting for phase measurements, described in [22], has an expanded uncertainty value of $0.0001^{\circ}$ at $52.1 \mathrm{~Hz}$, considering 38 acquired samples and a $S N R$ of $75 \mathrm{~dB}$, resulting from the Monte Carlo Analysis carried out by the authors of [22] (green dot in Figure 3).

The VNA operating over the frequency range of $500 \mathrm{~Hz}$ up to $125 \mathrm{GHz}$, according to the datasheet, and considering a power at the input port that minimises the phase uncertainty, provides an expanded phase uncertainty from $900 \mathrm{~Hz}$ to $120 \mathrm{GHz}$ of about $0.03^{\circ}$ [14], as plotted in cyan in Figure 3.

\subsection{Discussion}

By looking at Figure 3 and Table 2, the universal counter keeps the same amount of measurement uncertainty, higher than the other methods, until the frequency does not exceed the $10^{5} \mathrm{~Hz}$, from which a rapid increase of the uncertainty values can be observed. This trend is due to the limitations of the universal counter performance at high frequencies caused by the time resolution of 100 ps of the internal clock, and at low frequencies by the trigger jitter due to the low slope of the sinewave signal affected by noise.

The VNA exhibits a lower measurement uncertainty amplitude than the universal counter, maintaining the same uncertainty value at the higher frequencies. Moreover, the frequency range is higher than the universal counter one.

The PVC works in a frequency range that is smaller than the two previous instruments. It can be seen, however, that the uncertainty amplitude quickly decreases approaching the upper end of the frequency range to values lower than the previous instruments.

The interferometer operates at a higher frequency than the other instruments; however, the proposed prototype considered in the comparison can work within a narrow frequency range [17] and provides an uncertainty that is much higher than the VNA one. The implementation of an interferometer operating on a wide range of frequency represents, in fact, is still an unsolved problem.
The three-parameter sine fitting for phase measurement reaches the lower uncertainty amplitude of this comparison; however, this result is obtained at the unique frequency of $52.1 \mathrm{~Hz}$

In general, it must be noted that the instruments working within narrow frequency ranges or at a specific frequency exhibit the lowest measurement uncertainty (such as the ADC threeparameter sine fit, PVC, and interferometry). On the other hand, the universal counter and the VNA work within wide frequency ranges (lower and higher frequencies, respectively), but they exhibit higher uncertainties than narrow-band instruments. A single method that is able to work from few $\mathrm{MHz}$ to a few $\mathrm{gHz}$ with low uncertainty has not been published yet.

\section{CONCLUSIONS}

Several current applications require the measurement of the phase of sinewave signals, assuring high accuracy. Various methods and instruments have been developed for this purpose. However, it is not possible to find a method that is capable of covering a wide range of frequencies while also maintaining a low value of measurement uncertainty that can be used to develop a universal and highly accurate phase measurement instrument. In this article, an overview of the main methods used to measure the phase of a sinusoidal signal aimed at assuring high accuracy among the results is presented. Three main classes of methods have been identified, together with examples of their implementation in instruments available on the market as well as the scientific literature's proposals and prototypes. Then, a comparison of the instruments described in terms of the expanded uncertainty for the phase measurement of sinewave signal has been carried out.

Each of the considered cases requires an individual approach and to take into account the available features based on the technical data of the instrumentation used and the relevant measurement conditions for evaluation purposes. To that end, some useful information can be found in the comparison results reported in the paper.

This information can help in (i) finding the right method or instrument for a specific application by looking at the target uncertainty, (ii) designing new methods to improve phase measurement accuracy, and (iii) improving the existing ones in the covered frequency range while overcoming drawbacks as the requirement of isofrequential signals or the huge number of signal samples.

Table 2. Performance comparison in terms of expanded uncertainty of the instruments available on the literature for the measurement of the phase of sinewave signals.

\begin{tabular}{lccc}
\hline Method or instrument & Frequency range & Expanded uncertainty & Phase measurement method class \\
\hline $\begin{array}{l}\text { Keysight 53200A Series RF/universal frequency } \\
\text { counter/timers }\end{array}$ & $1 \mathrm{mHz}-350 \mathrm{MHz}$ & $\begin{array}{c}0.3^{\circ} \text { from } 1 \mathrm{mHz} \text { to } 100 \mathrm{kHz} \\
1.5^{\circ} \text { at } 10 \mathrm{MHz} \\
12.3^{\circ} \text { at } 100 \mathrm{MHz}\end{array}$ & Event-counting-based \\
& & $0.01^{\circ}$ at $7.7 \mathrm{~Hz}$ & $0.001^{\circ}$ at $77 \mathrm{~Hz}$, \\
PVC & $7 \mathrm{~Hz}-770 \mathrm{~Hz}$ & $0.0001^{\circ}$ at $770 \mathrm{~Hz}$ & Modulation-based \\
& & $0.37^{\circ}$ & Modulation-based \\
Interferometric Technique & $300 \mathrm{GHz}$ & $0.03^{\circ}$ & Modulation-based \\
VNA & $900 \mathrm{~Hz}-120 \mathrm{GHz}$ & $0.0001^{\circ}$ & Sampled-based \\
ADC 3-parameter sine fit & $52.1 \mathrm{~Hz}$ &
\end{tabular}




\section{ACKNOWLEDGEMENT}

The research presented in this paper has been supported from grant 70NANB15H167 'A Phase Measurement System for Calibrating Electroshock Weapon (PME)' awarded by the National Institute of Standards and Technology (NIST), USA.

The authors would like to thank Prof. Pasquale Daponte for his useful suggestions during all the phases of this research.

\section{REFERENCES}

[1] National Institute of Standards and Technology (NIST), Time and Frequency from A to Z. [Online]. Available: https://www.nist.gov/pml/time-and-frequencydivision/popular-links/time-frequency-z/time-and-frequency-zp\#phase

[2] K. Falaggis, D. P. Towers, C. E. Towers, Phase measurement through sinusoidal excitation with application to multi-wavelength interferometry, J. of Optics A: Pure and Applied Optics 11(5) (2009) pp. 1-11.

[3] A. Schicht, K. Huber, A. Ziroff, M. Willsch, L. P. Schmidt, Absolute phase-based distance measurement for industrial monitoring systems, IEEE Sensor Journal 9(9) (2009) pp. 10071013.

[4] A. Carta, N. Locci, C. Muscas, A PMU for the measurement of synchronized harmonic phasors in three-phase distribution network, IEEE Trans. On Instrumentation and Measurement 58(10) (2009) pp. 3723-3720.

[5] T. Y. Wu, Y. L. Lu, Error analysis and uncertainty estimation for a millimeter-wave phase-shift measurement system at $325 \mathrm{GHz}$, Measurement 59 (2015) pp. 198-204.

[6] N. G. Paulter, Jr., Method for measuring the phase spectrum of the output of a frequency source used in the calibration of an electroshock weapon characterization system, Journal of research of the National Institute of Standards and Technology 122(35) (2017) pp. 1-14.

[7] F. Picariello, I. Tudosa, L. D. Vito, S. Rapuano, N. G. Paulter, An initial hardware implementation of a new method for phase measurement of sinewave signals, Proc. of the 2019 11th International Symposium on Advanced Topics in Electrical Engineering (ATEE), 2019, Bucharest, Romania, pp. 1-6.

[8] T. S. Clement, P. D. Hale, D. F. Williams, C. M. Wang, A. Dienstfrey, D. A. Keenan, Calibration of sampling oscilloscopes with high-speed photodiodes, IEEE Transactions on Microwave Theory and Techniques 54(8) (2006) pp. 31733181.

[9] E. Balestrieri, L. De Vito, S. Rapuano, D. Slepicka, Estimating the uncertainty in the frequency domain characterization of digitizing waveform recorders, IEEE Trans. on Instrumentation and Measurement 61(6) (2012) pp. 1613-1624.

[10] D. R. Larson, N. G. Paulter, Some effects of temperature variation on sampling oscilloscopes and pulse generators, J. of Metrologia 43(1) (2006) pp. 121-128.

[11] G. Crotti, A. D. Femine, D. Gallo, D. Giordano, C. Landi, M. Luiso, Measurement of the absolute phase error of digitizers, IEEE Trans. on Instrumentation and Measurement 68(6) (2019) pp. 1724-1731.
[12] J. Dostal, V. Smotlacha, Dual interpolating counter architecture for atomic clock comparison, Proc. of the IEEE East-West Design \& Test Symposium (EWDTS 2014), 26-29 Sept., 2014, Kiev, Ukraine, pp. 1-4.

[13] Keysight Technologies, 53200A Series RF/Universal Frequency Counter/Timers, Datasheet. [Online]. Available: https://www.keysight.com

[14] Keysight Technologies, N5291A $900 \mathrm{~Hz}$ to $120 \mathrm{GHz}$ PNA mmWave System, Datasheet. [Online]. Available: https://www.keysight.com.

[15] A. De Marcellis, G. Ferri, E. Palange, A fully analog high performance automatic system for phase measurement of electrical and optical signals, IEEE Trans. on Instrumentation and Measurement 64(4) (2015) pp. 1043-1054.

[16] D. Owen, Good Practice Guide to Phase Noise Measurement, National Physical Laboratory, May 2004, ISSN 1368-6550.

[17] S. Hisatake, Y. Koda, R. Nakamura, N. Hamada, T. Nagatsuma, Terahertz balanced self-heterodyne spectrometer with SNRlimited phase-measurement sensitivity, Optics Express 23(20) (2015) pp. 26689-26695.

[18] S. Hisatake, G. Kitahara, K. Ajito, Y. Fukada, N. Yoshimoto, T. Nagatsuma, Phase-sensitive terahertz self-heterodyne system based on photodiode and low-temperature-grown GaAs photoconductor at $1.55 \mu \mathrm{m}$, IEEE Sensors Journal 13(1) (2013) pp. 31-36.

[19] S. Hisatake, J. Kim, K. Ajito, T. Nagatsuma, Self-heterodyne spectrometer using uni-traveling-carrier photodiodes for terahertz-wave generators and optoelectronic mixers, Journal of Lightwave Technology 32(20) (2014) pp. 3683-3689.

[20] T. Nagatsuma, S. Hisatake, M. Fujita, Hai Huy Nguyen Pham, K. Tsuruda,S. Kuwano, J. Terada, Millimeter-wave and terahertzwave applications enabled by photonics, IEEE Journal of Quantum Electronics 52(1) (2016) pp. 1-12.

[21] T. Nagatsumaet, S. Hisatake, Hai Huy Nguyen Pham, Photonics for millimeter-wave and terahertz sensing and measurement, IEICE Transactions on Electronics E99-C(22) (2016) pp. 173180.

[22] R. Lapuh, Phase estimation of asynchronously sampled signal using interpolated three-parameter sinewave fit technique, Proc. of the IEEE International Instrumentation and Measurement Technology Conference (I2MTC), 3-6 May, 2010, Austin, TX, pp. 82-86.

[23] IEEE Standard for Digitizing Waveform Recorders, IEEE Std 1057-2007, 2008. [Online]. Available: https://www.ieee.org

[24] E. Balestrieri, F. Picariello, S. Rapuano, I. Tudosa, Review on jitter terminology and definitions, Measurement 145 (2019) pp. 264273.

[25] E. Balestrieri, P. Daponte, L. De Vito, S. Rapuano, Jitter and its relatives: a critical overview, Proc. of the IEEE Instrumentation and Measurement Technology Conference, 2013, pp. 1141-1146.

[26] Understanding Key High-Performance Oscilloscope Specifications. [Online]. Available: https://www.tektronix.com/oscilloscopes

[27] A. Andreev, H. Klingbeil, D. Lens, Phase calibration of synchrotron RF signals, Proc. of 8th International Particle Accelerator Conference (IPAC2017), 2017, Copenhagen, Denmark, pp. 3945-3947. 